\title{
Ueber den Bau der Spinalganglien nebst Bemerkungen über die sympathischen Ganglienzellen.
}

Von

\author{
Dr. A. Schwalbe.
}

Hierzu Tafel IV.

Vorliegende Untersuchungen gingen zunächst davon aus, über die Anordnung der Ganglienzellen und Nervenfasern innerhalb der Spinalganglien, sowie über den Verlauf der letzteren und die Beziehung der sensibeln Wurzel zum Ganglion Klarheit zu erhalten, da gerade diese Verhältnisse bei den meisten früheren Untersuchungen wenig berücksichtigt waren. Natürlich konnte es dabei nicht ausbleiben, dass ich auch auf die feineren Structurverhältnisse der Ganglienzellen, auf die Art ihrer Verbindung mit den Nervenfasern und andere hierher gehörige Puncte meine Aufmerksamkeit richtete. Zugleich machte dies nöthig, auch die sympathischen Ganglienzellen zur Vergleichung heranzuziehen, da manche der streitigen Beobachtungen gerade an diesen zuerst gemacht worden waren. Immerhin blieb aber die Untersuchung des Faserverlaufs in den Spinalganglien meine Hauptaufgabe. Ich werde demnach mit meinen hierauf bezüglichen Untersuchungen beginnen und sodann Beobachtungen über die feinere Structur der Ganglienzellen, über ihren Zusammenhang mit den Nervenfasern und dergleichen folgen lassen.

Als Untersuchungsmaterial dienten mir Kalb, Schaf, Katze, Kaninchen, Meerschweinchen, Maus, Maulwurf, Taube, Eidechse und Frosch (sowohl Rana temporaria als Rana esculenta). Was die 
Dr. G. Schwalbe,

Spinalganglien der Fische betrifft, so habe ich auf eine eingehendere Untersuchung wegen der grossen Schwierigkeit hier in Bonn grössere Fische frisch in genügender Zahl zu erhalten, verzichtet.

An grösseren Spinalganglien vermögen wir die Structur nicht anders zu ermitteln, als durch in verschiedenen Richtungen durch das erhärtete Ganglion angelegte Schnitte. Zur Erhärtung kann man sich der bekannten Lösungen von Chromsäure und Kali bichromicum ${ }^{1}$ ) bedienen und die Schnitte mit Vortheil nachträglich mit Carmin färben. Allein diese Methode leistet hier nicht soviel, wie beim Rückenmark, indem das zähe Bindegewebe, welches in reichlichem Maasse die Ganglienzellenmassen durchsetzt, eine gleichmässige Erhärtung hindert. Anstatt durch Carmin habe ich nachträglich die Schnitte durch Ueberosmiumsäure gefärbt, indem eine 12-24stündige Einwirkung einer Ueberosmiumsäurelösung von $1 \%$ die so charakteristische blau-schwarze Färbung der markhaltigen Nervenfasern auch an Präparaten hervorruft, die mit Kali bichromicum behandelt waren. Es ist dies ein gutes Mittel, um den Faserverlauf innerhalb eines Spinalganglions zu erkennen. Noch besser eignet sich aber dazu das von F. E. Schulz e empfohlene Chlor-Palladium ${ }^{2}$ ), dessen wässrige Lösung bekanntlich fast ebenso wie Ueberosmiumsäure die markhaltigen Nervenfasern schwarz färbt, während es zugleich das Bindegewebe aufhellt und die Bindegewebskerne sowie die des Neurilemms gelb tingirt. Ich habe es mit grossem Vortheil zur Erhärtung von Spinalganglien des Kalbes benutzt, musste dabei aber bis zu einer Concentration von 1 auf 500 Theile Wasser hinaufgehen. Oefterer Wechsel der Flüssigkeit, in der sich immer flockige Niederschläge bilden, ist nöthig, um eine vollständige Erhärtung und Färbung zu erzielen. Wenn das Spinalganglion eine schwarzgraue Färbung angenommen hat, was nach den ersten 24 Stunden einzutreten ptlegt, ist es gewöhnlich zur Anfertigung von Schnitten geeignet. Sollte die Schnittfläche noch gelb gefärbt sein, so lasse man das Präparat noch einen Tag in der Lösung liegen und man wird nun sehr schöne Bilder erhalten, in denen das helle Bindegewebe sich

1) Vgl. Deiter's Untersuchungen über Gehirn und Rückenmark, herausgegeben von M. Schultze, pag. 21 .

2) Eine neve Methode der Erhärtung und Färbung thierischer Gewebe. Medic. Centralblatt 1867. Nr. 13. p. 193 und Der Ciliarmuskel des Menschen. Dieses Archiv Bd. III. 1867. p. $477 \mathrm{ff}$. 
scharf von den schwarzen Nervenfasern und gelbbraunen Nervenzellen abhebt. Ebenso deutliche, aber weniger elegante Präparate gibt die einfache Erhärtung der Spinalganglien erst in concentrirtem reinem Holzessig und nachher in Alkohol von 90\%. Man kann von so behandelten Ganglien mit Leichtigkeit die feinsten Schnitte machen und erkennt den Verlauf der Nervenfasern im hellen Bindegewebe aufs Deutlichste. Kleinere in Holzessig und Alkohol erhärtete Spinalganglien z. B. die des Kaninchens oder des Frosches legte ich behufs der Anfertigung von Schnitten zunächst in eine dickflüssige Gummizuckerlösung und entzog dieser das Wasser durch absoluten Alkohol. Frische Spiralganglien kleinerer Thiere werden durch die Behandlung mit ganz dünner Kali- oder Natronlauge soweit aufgehellt, dass man den Faserverlauf in denselben in toto bei schwachen Vergrösserungen übersehen kann, da die markhaltigen Fasern und die Ganglienzellen lange Zeit der Einwirkung dieses Rezgens widerstehen. Diese Methode gibt über manche Verhältnisse Aufschluss, die an Schnitten gar nicht zu ermitteln sind.

Die Gestalt eines Spinalganglions der Säugethiere ist nahezu halbmondförmig mit abgerundeten Spitzen, die Convexität nach der Peripherie kehrend, und platt. Der kleinste Durchmesser liegt in dorsoventraler Richtung, der grösste in der Richtung der Longitudinalaxe des Körpers, der dritte endlich in der Richtung von rechts nach links, also in transversaler Richtung. Den ersteren kann man als Dickendurchmesser, den zweiten, obgleich der grösste, als Breitendurchmesser und den letzteren als Längendurchmesser bezeichnen, indem dieser in der Richtung der ein- und austretenden Nervenfasern verläuft. Centralwärts ist die Ganglienmasse scharf von den eintretenden Nervenstämmchen abgesetzt, peripherisch dagegen findet ein allmähliger Uehergang der Ganglienmasse in den austretenden Nervenstamm Statt. Die eben beschriebenen Verhältnisse finden sich im Wesentlichen bei allen Säugethieren; bei anderen Wirbelthieren aber erleiden sie einige Modificationen. Wichtig ist in dieser Beziehung das Verhalten der Spinalganglien des Frosches. Ein solches bildet unter Zunahme des Dickendurchmessers und Abnahme des Breitendurchmessers eine $\mathrm{kugel}$ ige Anschwellung auf der hinteren Seite der sensibeln Wurzel. Aber auch diese Anschwellung dacht sich in peripherischer Richtung sanfter $a b$.

Betrachten wir die sensible Wurzel, so ergibt sich hier zunächst darin ein grosser Unterschied von der motorischen, dass wir dieselbe 
nicht mehr von der Ganglienmasse ohne Zerstörung des Ganglions trennen können, dass dieselbe äusserlich wenigstens mit jener ein Ganzes bildet. Bei den Säugethieren geht dies soweit, dass hier die sensible Wurzel ganz von Ganglienmasse umgeben wird. Zwar findet sich immer noch auch hier der Haupttheil derselben hinten, aber auch vorn bedecken sich nach und nach die einzelnen eintretenden Faserbündel mit derselben, was man schon mit unbewaffnetem Auge constatiren kann. Eine solche Betrachtung ergibt dann auch, dass sich an dieser vorderen Seite die einzelnen Faserbündel nicht alle in derselben Entfernung vom Rückenmark mit Ganglienmasse bedecken, sondern die einen früher, die anderen später; dem entsprechend sieht man denn auch an dieser Seite einige Faserbündel früher, andere später aus dem Ganglion austreten.

Wesentlich einfacher sind dagegen die Verhältnisse beim Frosch. Hier liegt die Ganglienmasse, obwohl mit der sensibeln Wurzel auch fest verbunden, doch nur dem hinteren Theile derselben an, während nach der Seite der motorischen Wurzel zu die Nervenfasern während ihres ganzen Verlaufes frei bleiben.

Soviel über die schon mit unbewaffnetem Auge erkennbaren Verhältnisse.

Ueber den Faserverlauf und die Natur der Ganglienzellen in den, Spinalganglien sind bisher besonders drei verschiedene Ansichten aufgestellt worden. Nach der einen, die besonders ron R. Wa g n e ${ }^{1}$ ) vertheidigt wurde, kommen in denselben nur bipolare Ganglienzellen vor, die einen Fortsatz central, den anderen peripherisch entsenden, so zwar, dass sich eine dieser Zellen in den Verlauf jeder sensibeln Faser einschaltet und gar keine sensible Faser ohne bipolare Ganglienzelle vorkommt, während die motorischen Fasern sich nicht an der Bildung des Spinalganglions betheiligen. Ewas modificirt wurde diese Ansicht durch Bid der ${ }^{2}$ ), welcher wie R. Wag ner vorzugsweise Fische untersuchte. Auch er nimmt nur bipolare Ganglienzellen in den Spinalganglien an mit der Modification jedoch, dass beide Fortsätze dicht neben einander entspringen und beide peripherisch verlaufen können.

1) Handwörterbuch der Physiologie. Bd. 3. Artikel: Sympathischer Nerv, Ganglienstructur und Nervenendigungen. p. 361 ff. und Neurologische Beobachtungen. Göttingen 1854. p. 8 ff.

2) Zur Lehre von dem Verhältniss der Ganglienkörper zu den Nervenfasern. Leipzig 1847. 
Ueb. d. Bau d.Spinalganglien n. Bemerk. ü. d. sympathischen Ganglienzellen. . 49

Eine wesentlich andere Ansicht stellte Axmann ${ }^{1}$ ) auf, der sich auf Grund seiner Untersuchungen am Frosch von der überwiegenden Existenz unipolarer Ganglienzellen in den Spinalganglien überzeugt hielt. Nach ihm gibt es einmal einfach durchtretende sensible und motorische Fasern, die innerhalb des Ganglions mit keinen Ganglienzellen in Verbindung stehen, sodann aus den Zellen der Spinalganglien entspringende Fasern, von ihm "gangliospinale« genannt. Diese verlaufen theils central sowohl in die motorischen als in die sensibeln Wurzeln, theils schliessen sie sich bündelweise den durchtretenden Fasern in peripherischer Richtung an. Axmann statuirt also eine gewisse Betheiligung auch der motorischen Wurzel an der Bildung des Spinalganglions.

Die dritte Ansicht und zugleich die einfachste ist die von Kölliker ${ }^{2}$ ). Nach ihm hat einmal die motorische Wurzel gar nichts mit dem Spinalganglion zu thun; aber auch die sensibeln Fasern ziehen einfach durch das Ganglion hindurch, ohne hier mit Ganglienzellen in Yerbindung zu stehen. Letztere, die aučh Kölliker für überwiegend unipolar hält, entsenden ihre Nervenfasern, von Kölliker "Ganglienfasern “ genannt, "in überwiegender Mehrzahl, vielleicht alle, peripherisch, “ und schliessen sich diese dann den durchtretenden sensibeln Fasern an. Ganz ähnlich, nur noch entschiedener äussert sich v. Baerensprung ${ }^{3}$ ) in einer den Anatomen wenig bekannt gewordenen Arbeit über den Zoster nach Untersuchungen an den Spinalganglien des Kindes. Er vergleicht den Bau eines Spinalganglions mit dem einer Drüse. Die einzelnen Ganglienkugeln gruppiren sich zu Läppchen, in deren Centrum sich die aus den einzelnen Zellen entspringenden Fasern zu einem Stämmchen sammeln, dem Ausführungsgange eines Drüsenläppchens vergleichbar, welches sich den durchtretenden Fasern in peripherischer Richtung anschliesst.

Man sieht also, dass es bei der Erörterung dieser Verhältnisse sich zunächst darum handelt, die Frage zu entscheiden: Kommen in den Spinalganglien der Wirbelthiere vorwiegend unipolare oder bi-

1) De gangliorum systematis structura penitiori eiusque functionibus. Berolini 1847.

2) Gewebelehre. 4. Auflage. p. 344.

3) Beiträge zur Kenntniss des Zoster. Annalen des Charité-Krankenhanses. XI. p. 96. 
Dr. G. Schwalbe,

polare Zellen vor? Bei den Fischen ist das Vorkommen bipolarer Zellen sicher constatirt. Dieselben scheinen hier den grössten Theil des Spinalganglions zu bilden. Anders ist es bei den übrigen Wirbelthieren. Nach den übereinstimmenden Untersuchungen aller neueren Forscher sind bei letztgenannten Thieren die Spinalganglienzellen unipolar. Mit diesem Resultate sind auch meine Beobachtungen im Einklang. Mir sind während einer langen Reihe von Untersuchungen nur zwei spinale Ganglienzellen von Säugethieren mit zwei Fortsätzen vorgekommen, die eine in einem Spinalganglion des Schafes, die andere im Ganglion Gasseri des Kalbes. In diesen beiden Fällen waren die Fortsätze nach einer Seite gerichtet und entsprangen dicht neben einander, etwa in der Weise, wie Bidder in seiner schon oben citirten Abhandlung dies auf Tafel I, Fig. 3 abbildet. Alle Zellen, die mir sonst bei Säugethieren, Vögeln, Reptilien und Amphibien zur Beobachtung kamen, zeigten nur e inen Fortsatz. Ich muss also die Unipolarität der Spinalganglien bęi den genannten Thieren für das Gewöhnliche halten.

Die Unipolarität der Zellen vorausgesetzt sind nun in Betreff des Faserverlaufs innerhalb der Spinalganglien drei Möglichkeiten denkbar: Entweder ziehen alle Ganglienzellenfortsätze nach der Peripherie oder 2) sie verlaufen alle central oder 3) es verlaufen nur einige central, während die anderen nach der Peripherie streben. In der Entscheidung dieser Frage beruht die Hauptschwierigkeit der Erkenntniss des Baues der Spinalganglien.

Gehen wir auf jeden der möglichen Fälle genauer ein, so können wir sogleich den zweiten als unhaltbar ausschliessen.

In der That ist nichts leichter zu zeigen, als dass nicht alle aus Ganglienzellen entspringenden Fasern central verlaufen können. Dies wurde schon vor mehr als zwanzig Jahren durch die genauen Zählungen der Fasern und durch die Messungen von Bidder und Volkmann ${ }^{1}$ ) widerlegt, indem diese Forscher zu dem Resultate kamen, dass viel mehr Nervenfasern aus dem Spinalganglion austreten, als mit der sensibeln Wurzel eintreten, ferner dass der austretende Nervenstamm sich dicker zeigt, als der eintretende. Auch ich kann die Richtigkeit dieser Thatsache bestätigen; natürlich sind Säugethiere, wenigstens die grösseren, ungeeignete Objecte zur Ent-

1) Die Selbständigkeit des sympathischen Nervensystems durch anatomische Untersuchungen nachgewiesen. Leipzig 1842. 
Ueb. d. Bau d. Spinalganglien n. Bemerk.ü. d. sympathischen Ganglienzellen. 51

scheidung dieser Frage; so haben denn auch Bidder und Volkmann ihre Zählungen und Messungen am Frosch vorgenommen. Ausser dem Frosch habe ich namentlich bei der Eidechse dieses Verhalten sehr deutlich ausgesprochen gefunden, und war hier die eintretende sensible Wurzel 0,149 Mm. breit, während der austretende Stamm beinahe das Doppelte maass, nämlich $0,249 \mathrm{Mm}$. Wenn man noch hinzurechnet, dass der letztere auch dicker, als der eintretende Stamm ist, so tritt der Unterschied noch schärfer hervor.

Bei Säugethieren lassen sich leider keine auch nur annähernd genauen Messungen anstellen, da die sensible Wurzel hier in mehrere Stämmchen zerspalten in das Ganglion eintritt. Dass nun die Dickenzunahme des austretenden Stammes nicht etwa auf einer reichlicheren Ansammlung von Bindegewebe, sondern nur auf Vermehrung der Nervenfasern beruht, lässt sich ebenfalls überall leicht constatiren, Es folgt demnach hieraus, dass in jedem Spinalganglion eine sehr beträchtliche Anzahl von Nervenfasern ihren Ursprung nimmt und peripherisch verlaufen muss. Damit ist denn auch die eine der drei oben aufgestellten Möglichkeiten, es möchten alle Fasern central verlaufen, widerlegt.

Es bleibt also nur noch übrig, darüber zu entscheiden, ob alle aus den Ganglienzellen entspringenden Fasern peripherisch oder ob auch einige central verlaufen. Letzteres ist, wie erwähnt, die Ansicht Axmann's. Wenn nun wirklich aus den Spinalganglienzellen entspringende Fasern central verlaufen sollten, so könnten dies offenbar nach den oben angeführten Thatsachen nur sehr wenige sein. Meiner Meinung nach verlaufen aber alle "gangliospinalen " Fasern peripherisch, und stütze ich mich dabei auf folgende Thatsachen.

Macht man durch ein Spinalganglion des Frosches einen Längsschnitt, so sieht man, wie Fig. 16, Taf. IV zeigt, die Ganglienzellenmasse an der sensibeln Wurzel einseitig anliegen; die Fasern der letzteren ziehen an jener vorbei und lassen nur hier und da einige versprengte Nervenzellen erkennen. Die Hauptganglienmasse setzt sich anfangs sehr scharf vom sensibeln Stamme ab und verschmilzt erst weiter peripherisch mit diesem zu einem einheitlichen Ganzen. Dies geschieht aber, wie man an dem Schnitte sehr gut sehen kann, nicht etwa dadurch, dass die gangliospinalen Fasern in die durchtretenden Faserbündel eintreten und zwischen diesen central verlaufen, sondern, wie man sieht, strahlen sämmtliche Nervenfasern in p e r i $\mathrm{p}$ h er is ch e r Richtung aus und schliessen sich einfach in ihrem weiteren Verlauf 
dem austretenden Stamme an. Noch überzeugender lässt sich der ausschliesslich peripherische Verlauf der gangliospinalen Fasern an den Spinalganglien der Eidechse nachweisen, welche man, nachdem man sie durch verdünnte Natronlauge aufgehellt hat, in toto unter dem Mikroskop betrachtet (Fig. 17). Auch hier ist der austretende Stamm viel breiter, auch hier schliessen sich die gangliospinalen Fasern in ausschliesslich peripherischer Richtung dem sensibeln Stamme an. Der Verlauf dieser Fasern ist nun aber ein nicht so einfacher, wie er, um die Deutlichkeit des Bildes nicht zu stören, in den beiden Figuren dargestellt ist. Es lassen sich im Verlauf derselben vielmehr zwei Abschnitte unterscheiden. Jede Faser ist von ihrem Ursprung aus der Ganglienzelle an, bis sie durch die Hauptmasse der anliegenden Nervenzellen hindurchgedrungen ist, zunächst eine sogenannte $u m w i n d e n d e$ Faser, $d$. h. sie biegt sich. in mannigfachen Krümmungen um die einzelnen Ganglienzellen herum, umwindet dieselben theilweise. Dies ist auch der Grund, wesshalb es so schwer hält, die Nervenzellen mit ihren Fasern auf weite Strecken zu isoliren; die mannigfach gekrümmten Fasern brechen sehr leicht ab. Auf dem zweiten Abschnitte ihres Weges kann man die Faser als ge rade bezeichnen; sie geht dann in ziemlich gerader Richtung peripherisch, um sich den durchtretenden sensibeln Fasern anzuschliessen.

Eine Folge des anfangs so mannigfach gewundenen Verlaufs der gangliospinalen Fasern ist es nun auch, dass man an den Spinalganglien der meisten Thiere Mühe hat, sich über ihren weiteren Verlauf zu orientiren: In dieser Beziehung ist gerade die Eidechse ein besonders günstiges Object. Die Kleinheit des Ganglions gestattet es hier, alle Verhältnisse mit genügender Klarheit zu übersehen. Man überzeugt sich, dass die umwindenden Fasern, soweit man sie überblicken kann, alle nach der Peripherie umbiegen. Ich habe mir die sorgfältigste Mühe gegeben, auch einmal eine central verlaufende Faser aufzufinden, aber nie ist mir dies gelungen. Immer stellte es sich heraus, wenn wirklich einmal eine Faser anfangs centra] verlief, dass sie bald umbog, also einen mit der Convexität nach dem Centrum gerichteten Bogen bildete und peripherisch den übrigen Fasern sich anschloss. Dies ist besonders deutlich zu sehen an den Spinalganglien des Meerschweinchens, die man nach Behandlung mit verdünnter Kalilauge ebenfalls noch ganz unter dem Mikroskop betrachten kann. 
Ueb. d. Bau d. Ganglienzellen n. Bemerk. ü. d. symnpathischen Gangtienzellen. 53

Wenn schon aus den oben angeführten Thatsachen mit grosser Wahrscheinlichkeit hervorgeht, dass alle gangliospinalen Fasern peripherisch verlaufen, so wird diese Wahrscheinlichkeit fast zur Gewissheit erhoben durch ein Experiment, dass man beim Frosch an dem grossen Spinalganglion des für die vordere Extremität bestimmten dicken Nervenstammes leicht ausführen kann. Fasst man diesen Nervenstamm mit einer fest schliessenden Pincette und zieht ihn stark an in der Richtung nach der Peripherie, so gelingt es oft, denselben aus dem Rückenmark herauszuziehen, und, was das Wichtigste in diesem Falle ist, es sitzt das Spinalganglion ganz intact, nur von seiner bindegewebigèn Hülle entblösst, noch dem Nervenstamme an. Wenn von den Spinalganglienzellen aus ein Theil der Fasern central verliefe, so würde bei jenem Verfahren offenbar das Ganglion verletzt werden müssen.

Es geht nun gewiss so viel aus dem Angeführten hervor, dass es jedenfalls eine gewungene, sich auf $\mathrm{ke}$ in e positive Beobachtungen stützende Annahme ist, zu behaupten, es verliefen gangliospinale Fasern central. Die einzig natürliche und durch alle Beobachtungen gestützte Annahme ist die, dass man im Spinalganglion zwei völlig getrennte Fasersysteme zu unterscheiden hat: 1) das System der durchtretenden sensibeln Fasern, 2) das System der aus den Spinalganglienzellen entspringenden Fasern, die ich nach dem Vorgange von A x mann als gangliospinale bezeichne, und dass letztere sämmtlich in peripherischer Richtung sich den ersteren anschliessen und früher oder später mit ihnen innig mischen zu einem gemeinsamen Nervenstamm.

Hiermit haben wir zugleich den Grundriss des Baues sämmtlicher Spinalganglien der Wirbelthiere von den Amphibien an aufwärts gegeben, und es bleibt uns nun noch übrig, zu zeigen, wie sich diese so einfachen Verhältnisse namentlich bei den grösseren Säugethieren $\mathrm{zu}$ so verworrenen Bildern compliciren, wie wir dies z. B. auf Längsschnitten der Spinalganglien des Kalbes sehen. Man kann drei Grade der Complication annehmen. Die einfachsten Verhältnisse finden sich bei den Amphibien und Reptilien. Frosch und Eidechse sind Beispiele dafür. Hier liegt die Ganglienmasse nur einseitig den sensiblen Fasern an; letztere weichen kaum von der geraden Richtung ab und nehmen desshalb nur sehr spärliche Ganglienzellen zwischen sich. Fig. 16 vom Frosch und Fig. 17 von der Eidechse veranschaulichen diese Verhältnisse. Die Ganglien der 
Eidechse unterscheiden sich nur darin von denen des Frosches, dass bei jenen der Breitendurchmesser die übrigen Durchmesser an Grösse übertrifft, während beim Frosch alle drei Durchmesser wesentlich gleich sind. Der einfache sensible Stamm bildet bei beiden Thieren e ine Masse und zerfällt nicht in mehrere gesondert eintretende sensible Bündel.

Wesentlich complicirter sind die Verhältnisse bei den kleineren Säugern. Hier treten immer mehrere (bis acht) gesonderte sensible Stämmchen ein. Zugleich liegen dieselben aber nicht bloss der einen Seite der Ganglienmasse an, sondern werden von allen Seiten von derselben umgeben, wie man auf Querschnitten z. B. von Spinalganglien des Kaninchens leicht erkennen kann. Doch liegen auf der hinteren oder dorsalen Seite immer mehr Ganglienzellen, als auf der vorderen oder ventralen. Sodann bleiben die durchtretenden Bündel innerhalb des Ganglions auch nicht zu einem Stamme vereinigt, sondern viele Fasern weichen seitwärts von der geraden Richtung ab, nehmen Ganglienzellen zwischen sich und vereinigen sich in ihrem weiteren peripherischen Verlauf entweder wieder mit dem Bündel, mit welchem sie eintraten, oder sie schliessen sich, um auszutreten, einem benachbarten Bündel an. Es kommen dadurch schon vielfach sich kreuzende Faserrichtungen zu Stande. Dazu kommt dann noch, dass auch die gangliospinalen Fasern hier einen complicirteren Verlauf haben und sich z. B. nicht immer dem benachbarten durchtretenden Bündel anschliessen, sondern ein entfernter liegendes aufsuchen, um mit diesem in peripherischer Richtung weiter zu verlaufen. Auf dem Querschnitt erscheint ein solches Ganglion jedoch noch sehr einfach. Derselbe ist elliptisch, und man erkennt längs einer Linie innerhalb desselben, welche mit der grossen Axe parallel verläuft, die Querschnitte der durchtretenden Nervenbündel, während die peripherischen Theile des Querschnitts überwiegend aus Ganglienzellen bestehen, zwischen denen man natürlich die zahlreichen umwindenden Fasern bemerkt.

Die höchste Complication erreichen die Verhältnisse bei den grösseren Säugethieren, von denen ich Kalb und Schaf untersuchte. Es begegnet uns hier namentlich auf dem Längsschnitt ein solches Fasergewirr, dass wir uns in der That gar nicht zurecht finden würden, hätten wir uns nicht zuvor an kleineren Spinalganglien orientirt. Man betrachte nur Fig. 20, welche einen Längs-Dickenschnitt durch ein Spinalganglion des Kalbes vorstellt. Man findet 
Ueb. d. Bau d. Spinalganglien n. Bemerk. ü. d. sympathischen Ganglienzellen. 55

hier sowohl quer und schief durchschnittene Faserzüge, als solche, welche innerhalb der Ebene des Schnittes in allen möglichen Richtungen verlaufen; die Ganglienzellen liegen bald haufenweise zusammen, bald zwischen die Faserzüge eingesprengt. Hier orientirt uns allein der Querschnitt. Er zeigt uns, dass ein solches Spinalganglion als ein Complex von Ganglien der zweiten vorhin beschriebenen Kategorie aufzufassen ist. Auch hier treten mehrere sensible Nervenstämmchen ein. Ich zählte z. B. beim Kalb sieben solcher grösserer Bündel. Aber jedes derselben zeigte sich schon vor dem Eintritt in das Ganglion auf dem Querschnitt als aus drei bis vier secundären Bündeln bestehend, die nun innerhalb des Ganglions ein jedes für sich die Verhältnisse wiederholen; die wir bei den Ganglien der zweiten Kategorie fanden. Ein jedes dieser Bündel umgibt sich gesondert mit Ganglienzellen, kann aber auch Fasern $\mathrm{zu}$ benachbarten oder entfernteren Bündeln schicken. Ebenso können die gangliospinalen Fasern entsprechend den oben geschilderten Verhältnissen den mannigfachsten Verlauf zeigen. Fig. 19 habe ich ein solches querdurchschnittenes Bündel mit seinen umgebenden Ganglienzellen möglichst getreu abgebildet. Man erkennt, dass hier ein solches Bündel wesentlich so gebaut ist, wie ein ganzes Spinalganglion der kleineren Säugethiere. Solcher "secundärer Ganglien ", wie man sie nennen könnte, findet man nun auf dem Querschnitt gegen zwanzig; dazwischen kommen noch grössere oder kleinere Nervenbündel vor, die nur wenige oder keine Nervenzellen führen. Dies kommt daher, dass nicht alle Nervenstämmchen sich gleichzeitig mit Ganglienzellen mischen, sondern die einen früher, die anderen später; erstere zeigen sich dann auch wieder früher vollkommen frei von denselben.

Bei einem solchen Gewirr von Ganglienzellen und Nervenfasern scheint es fast unmöglich, sich zu überzeugen, dass auch hier die gangliospinalen Fasern peripherisch ziehen. Indessen gelingt es doch zuweilen durch Abschälen einiger besonders hervorspringender Ganglienkörnchen Bilder zu erhalten, wie Fig. 18. Es stammt dies Präparat vom Kalbe und wurde, um den Faserverlauf besser zu erkennen, wieder mit verdünnter Kalilauge behandelt. Man nimmt auch hier ohne Mühe denselben Bau wahr, wie er sich bei kleineren Thieren so leicht erkennen lässt; $a$ ist ein durchtretendes Stämmchen, b dasselbe weiter nach der Peripherie zu, nachdem es sich durch die aus den anliegenden Ganglienzellen entspringenden Fasern verstärkt' hat. Der verschlungene Verlauf der gangliospinalen Fasern ist hier angedeutet. 
Indem ich nunmehr zum zweiten Theil meiner Aufgabe, nämlich zur Anführung meiner auf die feinere Structur der Ganglienzellen bezüglichen Beobachtungen übergehe, hätte ich zuerst des von Fraentzel ${ }^{1}$ ) kürzlich beschriebenen "Epithels der Ganglienz elle a c zu gedenken. Ich kann hier, was die Thatsachen anbetrifft, einfach auf die vollkommen richtige Beschreibung von Fraentzel verweisen. Auch mir ist es gelungen, durch Anwendung der von Fraentzel empfohlenen Methoden mich von der Existenz einer epithelartigen Auskleidung der Ganglienzellenscheiden zu überzeugen, und muss ich noch besonders hervorheben, dass schon die Kerne derselben sich anders verhalten, als die des umgebenden Bindegewebes; denn während letztere elliptisch sind, zeigen sich erstere immer als runde Scheiben und stehen viel dichter, als die des interstitiellen Bindegewebes. Besonders deutliche Bilder erhielt ich bei Anwendung der kürzlich von Gerlach angegebenen modificirten Cohnheim'schen Goldchlorid-Methode.

Bei allen diesen Methoden muss man sich aber davor hüten, dass man nicht die Zeichnungen, welche durch das zackige Einschrumpfen der Ganglienzellen entstehen, mit dem epithelialen Belag verwechselt. Es können dadurch oft täuschend zellenähnliche Zeichnungen vorgespiegelt werden, in denen dann auch runde Kerne erscheinen können. Bei genauer Einstellung überzeugt man sich aber davon, dass die Kerne nur scheinbar innerhalb dieser Zeichnungen liegen, dass sie in Wahrheit zum wirklichen epithelartigen Belag gehören. Während nämlich in Folge der Einwirkung des doppeltchromsauren Kalis die Ganglienzellen schrumpfen, bleibt der Zellenbelag an der Scheide unmittelbar anliegen, und dies entspricht auch vollkommen den Verhältnissen im frischen Zustande. Denn niemals gelingt es, eine Ganglienzelle aus einem Spinalganglion eines Säugethiers mit Fortsatz und Scheide zugleich glatt zu isoliren. Immer erhält man hüllenlose Nervenzellen, auf denen freilich noch Reste der Scheide und des anstossenden Bindegewebes haften können. Im Ganzen ist Letzteres jedoch selten.

Wenn ich mich somit in allen wesentlichen Puncten der Beschreibung von Fraentzel anschliesse, so kann ich ihm doch nicht darin beistimmen, dass er diese Bildungen als "Epithel der Gang-

1) Beitrag zur Kenntniss von der Structur der spinalen und sympathischen Ganglienzellen. Virchow's Archiv. Bd. 38. 
Ueb. d. Bau d. Spinalganglien n. Bemerk. ü. d. sympathischen Ganglienzellen. $5 \pi$

lienzellen " bezeichnet. Meiner Meinung nach ist dies sogenannte Epithel in eine Kategorie zu bringen mit dem "Epithel« der Blutcapillaren und Lymphgefässe, welches von $\mathrm{H}$ is mit Recht von den eigentlichen Epithelien unterschieden und mit dem besonderen Namen des Endothels belegt wird. Ich verweise in dieser Beziehung auf die Auseinandersetzungen in der bezüglichen Arbeit von $\mathrm{H}$ is ${ }^{1}$ ). Wir haben es hier also mit wesentlich end otheli al en Bildungen zu thun. Die Scheide der Ganglienkugel besteht lediglich aus endothelialen Plättchen, die sich aber innig dem begrenzenden Bindegewebe anlegen, da sie nur eine Grenzschicht desselben darstellen. Diese Auffassung scheint auch Kölliker ${ }^{2}$ ) zu theilen, indem er die betreffenden Zellen ebenfalls mit denen der Capillaren vergleicht. Auch ihm gelang es, an den Ganglienzellen der Spinalganglien der Säugethiere sich vom Vorhandensein dieses zelligen Belags zu überzeugen.

Die eben besprochenen Verhältnisse liessen sich nun bei allen Säugethieren, die ich untersuchte, und auch bei den Vögeln, von denen ich freilich nur die Taube mit in den Kreis meiner Untersuchung gezogen habe, sowohl an den sympathischen, als spinalen Ganglienzellen mit Leichtigkeit nachweisen. Nur in der Zahl und Grösse der runden Endothelkerne im Umkreise einer Ganglienzelle fanden sich Unterschiede, wie sie eben durch die ungleiche Grösse der Nervenzellen bei den verschiedenen Thieren bedingt sein mussten. Wesentlich anders verhält sich aber die Sache beim Frosch. Während sich nämlich, wie schon erwähnt, bei den höheren Wirbelthieren die Ganglienzellen sowohl des Sympathicus als der Spinalganglien stets nur ohne Hülle isoliren lassen, isoliren sich die des Frosches wenigstens eben so leicht mit Hülle, als ohne dieselbe. Es scheint hier also der Zusammenhang der innersten Ganglienzellenscheide mit dem anstossenden Bindegewebe ein nicht so inniger zu sein, als bei den Vögeln und Säugethieren. Dies ist jedoch nicht der einzige Unterschied. Die zarte Hülle lässt beim Frosch keinen Zerfall in epithelähnliche Plättchen erkennen und enthält nur sparsame Kerne, die zuweilen, und zwar an den sympathischen Zellen häufiger, als an den spinalen, ganz fehlen können. Dies gilt jedoch für die Scheide nur so weit, als sie die Ganglienzelle selbst überzieht. Beim Uebergang in die Scheide der Nervenfaser finden sich auch hier

1) Ueber die Häute und Höhlen des Körpers. Basel 1865.

2) Gewebelehre. 5. Aufl. 1867. p. 251. 
Bilder, die vielleicht auf eine endotheliale Bildung zu deuten sind. Kollmann und Arnstein ${ }^{1}$ ) erwähnen bei Besprechung der Art und Weise des Abgangs der Spiralfaser vom Körper der Ganglienzelle eine feinkörnige Masse mit Kernen am Grunde der Zelle, da wo die gerade Faser eintritt, und bilden dieselbe auch an einigen Zellen ab. Sie glauben aber, dass diese Kerne der sich hier entwickelnden und zahlreiche Touren beschreibenden Spiralfaser zulkommen. Wenn nun auch in der That offer nur wenige quergestellte Kerne hier vorkommen, sodass man sich an jene Forscher anschliessen könnte, so findet man doch andererseits nicht selten eine reichliche Kernbildung, die ziemlich weit den Stiel herabreichen kann, wie Fig. 8 zeigt, welche eine derartige sympathische Ganglienzelle vom Frosch darstellt. Die Kerne stehen hier ziemlich regelmässig und dicht beisammen, so dicht, dass man in diesem Falle kaum die Art und Weise des Eintritts der geraden Nervenfaser in die Ganglienzelle erkennen konnte. Die Kerne sind zugleich etwas eckig, was aber möglichenfalls der Einwirkung einer dünnen Chromsäurelösung, womit die Zelle behandelt wurde, zuzuschreiben ist. Wenn man solche Bilder sieht, ist man in der That versucht, sie mit dem Befunde der Ganglienzellenscheiden bei den Sängethieren zu parallelisiren. Nur ist es mir nicht gelungen, deutliche Zellengrenzen zwischen den Kernen nachzuweisen. Allein dies gelingt bei manchen Säugethieren auch nicht mehr, trotzdem dass die Anordnung der Kerne sonst vollständig der beim Kalbe und Schafe gleicht. Wir können demnach nicht anstehen, diese Bildungen für Andeutungen eines Epithels zu halten, wie dies auch schon Fraentzel ${ }^{2}$ ) andeutet.

Schliesslich muss ich noch anführen, dass ausser Robin und R. Wag ner, die Fraentzel citirt, auch noch $R e \mathrm{mak}^{3}$ ) in seinen Schriften ein "Ganglienzellenepithel« erwähnt. Er sagt nämlich von der Scheide der sympathischen Ganglienzellen: "Die Scheide der letzteren besteht, wie in den Spinalganglien, aus einer weichen Zellenschi cht und einer festen Membran." Deutliche Abbildungen der runden endothelialen Kerne finden sich sodann in einer älteren Abhandlung von Valentin ${ }^{4}$ ) und in der allgemeinen Anatomie von

1) Die Ganglienzellen des Sympathicus. Zeitschr. f. Biologie. Bd. II. p. 285.

2) 1. c. p. 9 des Separatabdrucks.

3) Ueber multipolare Ganglienzellen. p. 3. des Separatabdrucks.

4) Ueber die Scheiden der Ganglienkugeln und deren Fortsetzungen. Mül ler's Archiv 1839. p. 145. 
Ueb. d. Bau d. Spinalganglien n. Bemerk. ü. d. sympathischen Ganglienzellen. $\quad 59$

Henle ${ }^{1}$ ). Endlich finden sich sogar über die Entwicklung dieser Gebilde schon Angaben in der Dissertation von $\mathrm{Schram} \mathrm{m}{ }^{2}$ ), der die innere Ganglienzellenscheide aus spindelförmigen Zellen entstehen lässt, die später zu Plattenzellen auswachsen.

Ich kann jetzt zur Besprechung der Ganglienzellen selbst übergehen, die ich meist frisch in Jodserum untersuchte; doch habe ich auch die verschiedensten anderen Isolationsmethoden versucht, ohne dabei wesentliche Vortheile zu erzielen. Nur an Schnitten, die frisch in Ueberosmiumsäure von $1 / 4-1 / 2 \%$ gelegt wurden, lassen sich die einzelnen Zellen ebenso intact erhalten, wie in Jodserum und dabei leicht isoliren.

Eine fibrilläre Structur, wie sie M. Schultze ${ }^{3}$ ) and Deiter $\mathrm{S}^{4}$ ) von den centralen Ganglienzellen beschreiben, lässt sich auch an manchen spinalen und sympathischen gegen den Austritt der Nervenfasern hin erkennen, am besten an den multipolaren Zellen des Säugethier-Sympathicus. In einer kürzlich erschienenen Arbeit bespricht Jolly ${ }^{5}$ ) diese fibrilläre Structur der centralen Ganglienzellen und glaubt, dass sie eine Folge der Einwirkung der dünnen Chromsäure-Lösungen sei. Alles, was im frischen Zustande körnig oder gestrichelt erscheint, will er lieber auf eine Unregelmässigkeit der Oberfläche, als auf eine weitere Differenzirung des Inhalts deuten. Diese Deutung ist wohl etwas künstlich; auch lässt sich sehr leicht nachweisen, dass jene körnigen und gestrichelten Partieen in der That in der Substanz der Ganglienzelle liegen. Wenn die Strichelung durch die Einwirkung dünner Chromsäure-Lösungen deutlicher wird, so liegt hier dieselbe Wirkung vor, wie z. B. bei der Behandlung der marklosen Nervenfasern mit demselben Reagens, in Folge dessen bekanntlich hier die fibrilläre Structur sehr deutlich wird. Meiner Meinung nach müssen wir also auch in jener feinen Strichelung eine eigenthümliche Anordnung der Ganglienzellsubstanz erkennen.

Während nun diese fibrilläre Strichelung am Abgang der Fort-

1) Tafel IV Fig. $7 \mathrm{~A}$.

1864. p. 12.

2) Neue Untersuchungen über den Bau der Spinalganglien. Würzburg

3) 1. c. p. $X \nabla$.

4) l. c. p. 58.

5) Ueber die Gang!ienzellen des Rückenmarks. Zeitschr. f. wissensch. Zoologie 1867. 
sätze in den multipolaren Zellen die gewöhnlichste ist, findet sich eine andere Art der Differenzirung der Substanz besonders in den Zellen der Spinalganglien. Dieselbe ist hier nämlich mehr oder weniger deutlich concentrisch um den Kern angeordnet. Von Wichtigkeit ist, dass sich dies Verhältniss auch im ganz frischen Zustande nachweisen lässt; doch zeigen nicht alle Zellen dasselbe gleich deutlich. 'Am deutlichsten zeigt sich diese Anordnung bei gewissen wirbellosen Thieren. So erwähnt Le yd i g ${ }^{1}$ ) dieselbe von den Ganglienkugeln des Dyticus, der Locusta, des Blutegels; W alter ${ }^{2}$ ) beschreibt sie vom Flusskrebs und Limnaeus stagnalis. Auch ich habe bei den wirbellosen Thieren diese Verhältnisse am deutlichsten gefunden und kann in dieser Beziehung namentlich die grossen Zellen des unteren Schlundganglions von Limax empiricorum empfehlen, von denen ich in Fig. 15 eine Abbildung gebe, an welcher man die concentrische Strichelung sehr deutlich erkennt. Das Ganglion wurde frisch in Jodserum untersucht.

Es sei mir hier gestattet, noch kurz einer auffallenden Erscheinung zu gedenken, die ich einmal an einigen Spinalganglienzellen des Maulwurfs fand. Ich bemerkte nämlich in denselben mehrere helle elliptische Räume, die neben dem deutlich sichtbaren Kern in der Zelle lagen und sich bei genauerer Betrachtung als Vacuolen zu erkennen gaben, wofür der helle wasserklare Inhalt mit dem für Vacuolen so charakteristischen mattröthlichen Aussehen sprachen. In Fig. $4 \mathrm{~B}$ ist eine solche Zelle mit drei Vacuolen abgebildet. $\mathrm{Ob}$ dieselben in den Ganglienzellen beim Maulwurf eine weitere Verbreitung haben, muss ich unentschieden lassen. Möglich ist es auch, dass ich es hier mit einer Leichenerscheinung zu thun hatte, da das betreffende Thier nicht mehr ganz frisch war. Aber selbst dann bleibt diese Vacuolen-Bildung eine eigenthümliche Erscheinung, die meines Wissens sonst noch nicht beobachtet ist.

Was ferner den Kern der Ganglienzelle betrifft, so habe auch ich öfter eine radiäre Anordnung seiner Substanz, wie sie Kölliker ${ }^{3}$ ) beschreibt, constatiren können, und zwar nimmt dieselbe hier das Kernkörperchen zum Mittelpuncte. Dass aber diese Streifung nicht

1) Vom Bau des thierischen Körpers. p. 85.

2) Mikroskôpische Studien über das Centralnervensystem wirbelloser Thiere. Bonn 1863.

3) l. c. p. 254 . 
eb. d. Bau d. Spinalganglien n. Bemerk. ü. d. sympathischen Ganglienzellen. 61

in Zusammenhang zu bringen ist mit Fasern, die vom Kernkörperchen ausgehend zu Nervenfasern werden, wie dies Frommann ${ }^{1}$ ) annimmt, muss ich schon hier ausdrücklich betonen.

Das Vorkommen $z$ wei er Kerne ist, abgesehen von den sympathischen Zellen des Kaninchens und Meerschweinchens, die ich sogleich besprechen werde, wohl äusserst selten. Zwar sagt Kölli k e r ${ }^{9}$ ), Nervenzellen mit mehrfachen Kernen seien bei jungen Thieren häufig, bei erwachsenen dagegen sehr selten; allein meiner Meinung nach sind dieselben auch bei jungen Thieren selten genug. Ich habe gerade das Kalb auf diese Verhältnisse genau untersucht und habe nie das Glück gehabt, eine zweikernige Zelle zu finden. Auch Rindsembryonen, die mir von verschiedenen Entwicklungsstadien zu Gebote standen, zeigten in ihren Ganglienzellen immer nur einen Kern. Es ist desshalb wohl auch noch etwas verfrüht, Theilungen der Ganglienzellen wegen des öfteren Vorkommens zweier Kerne anzunehmen, zumal die Verhältnisse beim Kaninchen, auf die ich nunmehr übergehe, auf einen wesentlich anderen Entwicklungsmodus hinweisen.

Das Vorkommen dieser zweikernigen Zellen im Sympathicus des Kaninchens wurde neuerdings von $\mathrm{Guye}^{3}$ ) besonders hervorgehoben, obgleich derselbe nicht der erste ist, welcher dieselben beschreibt. Dies ist vielmehr Remak, der auf Tafel II Fig. 15 seiner Observationes anatomicae et microscopicae de systematis nervosi structura (Berolini 1838) von den Zellen des Kaninchensympathicus schon sehr deutliche, freilich bei schwacher Vergrösserung entworfene Abbildungen gibt und in seiner Beschreibung auch des Vorkommens zweier Kerne gedenkt. Während nun $\mathrm{Remak}$ die betreffenden Zellen mit mehreren Fortsätzen zeichnet, spricht Gu ye nur von bipolaren Zellen. Ich kann mich nur Remak anschliessen, indem ich gefunden habe, dass man bei sorgfältiger Isolirung hier immer schöne multipolare Zellen erhält, deren Fortsätze jedoch leider nur auf kurze Strecken zu erhalten sind und auch leicht schon am Zellenkörper selbst abreissen. Es gibt kaum ein zierlicheres Bild, als diese feinkörnigen vielstrahligen, mit je zwei grossen klaren runden oder ellipsoidischen Kernen ausgestatteten Ganglienzellen. Jeder

1) Virchow's Archiv. Bd. 31 .

2) 1. c. p: 255 .

3) Die Ganglienzellen des Sympathicus beim Kaninchen. Med. Central blatt 1866. N. 56. p. 881 . 
Kern kann zwei oder mehr Kernkörperchen enthalten. Eine grössere Zahl derselben ist hier ziemlich häufig. Nicht richtig ist es, wenn Guye immer nur von bipolaren Ganglienzellen analog denen der Fische redet. Man erhält bei der Isolation zwar oft nur zwei Fortsätze, ebenso oft aber mehr, bis sechs, und in ersterem Falle sieht man immer deutlich, dass Fortsätze abgerissen sind. Ich gebe in Fig. 10, 11 und 12 Abbildungen mehr oder weniger intact erhaltener Zellen, die die betreffenden Verhältnisse erläutern. Gu y e lässt ferner jeden seiner Forsätze mit einem Kern zusammenhängen und bezeichnet demgemäss letztere als "Kernplatten". Ich habe nie trotz der sorgfältigsten darauf verwendeten Untersuchung sowohl im frischen Zustande, als nach Anwendung der verschiedensten Reagentien etwas Derartiges finden können.

Bei erwachsenen Kaninchen finden sich im Sympathicus nur zweikernige Zellen mit Ausnahme einiger weniger einkerniger wirklich bipolarer Zellen im St a m m des Grenzstranges, die mit Re mak'schen Fasern in Verbindung stehen. Diese Zellen sind äusserst blass und zart, sehr vergänglich und entgehen leicht der Beobachtung, zumal sie auch bedeutend kleiner sind, als die zweikernigen. Es gelang mir einmal, sie gut $\mathrm{zu}$ isoliren und ihren Zusammenhang an beiden Polen mit Remak'schen Fasern unzweifelhaft nachzuweisen. Am Uebergang einer solchen Faser in die Zelle findet sich immer eine feinkörnige, mehrere dunkle, scharf contourirte, runde Kerne enthaltende Anschwellung (Fig. 13). Während dies nun die einzigen einkernigen Zellen sind, die im Sympathicus erwachsener Kaninchen vorkommen, und diese noch dazu auf den Stamm beschränkt sind, finden sich in den sympathischen Ganglien junger Thiere ausser den gewöhnlichen zweikernigen multipolaren Zellen noch zahlreiche einkernige, welche sich aber nur durch den Mangel des zweiten Kernes von jenen unterscheiden; nur sind sie etwas zarter und lassen sich schwerer isoliren. Ich habe desshalb auch noch nicht die Anzahl ihrer Fortsätze sicher ermitteln können, glaube aber, dass auch diese Zellen multipolar sind: Soviel scheint nun aber aus diesen allerdings unvollständigen Angaben hervorzugehen, dass sich im Sympathicus des Kaninchens zweikernige Zellen erst aus einkernigen entwickeln und dass also erstere hier nicht auf beginnende Zellentheilung zu deuten sind. Was die Art der Entstehung der zweikernigen aus den einkernigen Nervenzellen betrifft, so könnten dieselben entweder durch einfache Theilung des ursprünglich einfachen Kernes entstehen, oder 
es könnten zwei einkernige Zellen zu einer zweikernigen verschmelzen. Welche von diesen Annahmen die richtige ist, müssen künftige Untersuchungen lehren.

Ich habe nun im Sympathicus der verschiedensten Thiere nach zweikernigen Zellen gesucht und habe bis jetzt nur noch beim Meerschweinchen deren gefunden. In der That gleichen hier die Verhältnisse ganz den beim Kaninchen beschriebenen. In Fig. 14 gebe ich eine Abbildung von einer multipolaren zweikernigen Zelle aus dem Sympathicus des Meerschweinchens, deren Kerne resp. drei und zwei Kernkörperchen enthalten. Möglich ist es, dass man noch bei anderen Nagern Aehnliches beobachtet.

Was endlich das Kernkörperchen betrifft, so will ich nur darauf aufmerksam machen, dass dasselbe öfter, als man wohl bisher annahm, zu zweien in einem Kerne vorkommt. So findet man namentlich bei jungen Kälbern oft die Hälfte der Zellen mit zwei Kernkörperchen. Auch mehr als zwei derselben sind keine Seltenheit (Fig. 3 und Fig. 14). In diesem Falle findet man öfter, dass die Kernkörperchen keine kugelrunde, sondern eine eckige Gestalt besitzen und sich sehr in ihrer Grösse unterscheiden. Körner im Kernkörperchen sind nicht selten. Ich kann denselben aber keine weitere Bedeutung zuschreiben, am wenigsten, wie Frommann, dieselben für optische Querschnitte feinster Kernkörperchenfäden halten. Ich erkläre sie einfach für dichtere Stellen innerhalb der compacten Kernkörperchensubstanz; wenigstens konnte ich bei den von mir untersuchten Wirbelthieren darin keine Vacuolen erkennen, was jedoch im unteren Schlundganglion von Arion empiricorum sehr leicht gelang. Hier besitzen die Ganglienzellen sehr grosse feingranulirte Kerne, die bis gegen acht ausgebildete runde Kernkörperchen enthalten können, deren jedes in seinem Centrum eine helle Vacuole birgt (Fig. 15). Die Vacuole wächst mit der Grösse der Kernkörperchen. Letztere scheinen hier durch partielle Verdichtung der Kernsubstanz zu entstehen. Wenigstens sieht man ausser den deutlich als solche zu erkennenden Nucleolis mehrere dunkle Puncte in der Substanz des Nucleus und zwar von verschiedener Grösse, sodass man von den kleinsten Körnern bis zum kleinsten deutlich ausgeprägten Nucleolus alle Uebergänge beobachten kann. Die Vacuolenbildung findet dann im Laufe des Wachsthums dieser Gebilde statt.

Eine eingehendere Besprechung verdienen die zuerst von $\mathrm{Har}$ - 
less ${ }^{1}$ ), Lieberkühn ${ }^{2}$ ), Axmann ${ }^{3}$ ) und G. Wagener ${ }^{4}$ ), sodann neuerdings wieder von A rnold ${ }^{5}$ ) gemachten Angaben über den Zusammenhang des Axencylinders der "geraden Faser “ mit dem Kernkörperchen der Ganglienzelle, für welche in neuester Zeit Kollmann und Arnstein ${ }^{6}$ ), Guye ${ }^{7}$ ), Bidder ${ }^{8}$ ) und Jolly ${ }^{9}$ ) sich günstig aussprachen. Hieran muss sich dann die Besprechung des von Arnold und Courvoisier ${ }^{10}$ ) beschriebenen Netzes vom Kernkörperchen ausgehender feiner Fasern, sowie der von Arnold und Beale ${ }^{11}$ ) entdeckten Spiralfasern schliessen.

Ueber die hierher gehörigen Angaben Guy e's habe ich schon oben mein Urtheil abgegeben. Was die Angaben Jolly's betrifft, so bestätigt derselbe im Wesentlichen die Beobachtungen von $\mathrm{Dei}$ ters, während er die von Frommann beschriebenen Kernröhren und Kernkörperchenfasern, ebenso wie M. Schultze, nicht finden konnte. Dagegen hat er in einigen wenigen Fällen einen Fortsatz des Kernkörperchens gesehen; doch gelang es ihm nie, denselben durch die Zellensubstanz hindurch bis zum Axencylinderfortsatz zu verfolgen. Jolly's Angaben sind demnach ebenfalls nicht dazu angethan, den Zusammenhang zwischen Axencylinderfortsatz und Kernkörperchen $\mathrm{zu}$ beweisen, abgesehen davon, dass die hierher bezüglichen Bilder nur in sehr seltenen Fällen erhalten wurden.

G. W a g e ner hat seine Beobachtungen hauptsächlich an wirbellosen Thieren angestellt. Ich habe in dieser Beziehung die grossen Ganglienzellen des unteren Schlundganglions von Arion empiricorum geprüft, habe aber nie dergleichen sehen können, trotzdem dass ich

1) Briefliche Mittheilung über die Ganglienkageln der Lobi electrici von Torpedo Galvanii. Müller's Archiv 1846. p. 283.

2) De structura gangliorum penitiori. Berolini 1849.

3) 1. c.

4) Ueber den Zusammenhang des Kernes und Kernkörpers der Ganglienzelle mit dem Nervenfaden. Zeitschrift f. wissensch. Zoologie. Bd. VIII. p. 455.

5) Virchow's Archiv. Bd. 32. p. 6.

$\begin{array}{ll}\text { 6) } 1 . \mathrm{c} . & \text { 7) } 1 . \mathrm{c.}\end{array}$

8) Weitere Untersuchungen über die Glandula submaxillaris des Hun-

des. Archiv ron Reichert und du B ois-Reymond 1867. p. 1.

9) $1 . \mathrm{c}$.

10) Dieses Archiv. Bd. II 1866. p. 13 ff.

11) Philosophical Transactions 1863. Part. II. 
Ueb. d.Bau d. Spinalganglien n. Bemerk. üb. d. sympathischen Ganglienzellen. 65

die Theile in ganz frischem Zustande und möglichst vor Druck geschützt untersuchte. Ich konnte vielmehr immer einen directen Uebergang der Substanz der eintretenden platten breiten Nervenfaser in die eigentliche concentrisch geschichtete Substanz der Ganglienzelle constatiren. Ebenso äussert sich auch $\mathrm{Buchholz}{ }^{1}$ ), der höchst sorgfältige Untersuchungen an Limnaeus stagnalis und Planorbis corneus anstellte. Er hält die Substanz der Nervenfaser für vollkommen identisch mit der der Ganglienzelle.

Die meisten der genannten Beobachter haben num aber die sympathischen und spinalen Zellen der Wirbelthiere und namentlich die des Frosches untersucht. Gerade hier finden sich aber die widersprechendsten Angaben. Während nämlich einige Forscher, wie Axmann, Frommann (Virchow's Archiv, Band 31, Taf. VI Fig. 10) und Bidder ${ }^{2}$ ) die gerade Faser mit dem Kern sich verbinden lassen, so dass ihre Contouren unmittelbar in die des Kernes übergehen, beschreiben die anderen einen Zusammenhang derselben mit dem Kernkörperchen. Was die wenigen Beobachtungen über den Zusammenhang des Ke r n es mit dem Axencylinder betrifft, so glaube ich Einiges zur Erklärung derselben beitragen zu können. Bei der Untersuchung von Spinalganglien, die einen Tag in Jodserum gelegen hatten, fand ich oft auffallend stark seitlich comprimirte Kerne, deren Contour an dem einen Pole des elliptischen Umrisses fehlte. Es schien in diesen Fällen vom Kern ein Fortsatz auszugehen, der aber nicht weiter durch die Substanz der Zelle hindurch verfolgt werden konnte. Noch überraschender wurde das Bild, wenn in der Richtung dieses Kernzipfels eine Nervenfaser von der Zelle abging. In manchen Fällen war auch wohl der Kern zu einer feinen Spitze ausgezogen, die sich in der Zellsubstanz verlor. In diesen Fällen fehlte aber das Kernkörperchen, das sonst immer noch als scharf umschriebenes Gebilde innerhalb des Kerns sichtbar war. So sehr ich nun anfangs gewünscht hatte, die Angaben der genannten Forscher wenigstens in Betreff des Zusammenhanges der Nervenfaser mit dem Kern bestätigen zu können, so konnte ich doch bei sorgfältiger Prüfung dieser Bilder nicht umhin, dieselben auf andere

1) Bemerkungen über den histologischen Bau des Centralnervensystems der Süsswassermollusken. Archiv v. Reichert $u, d u$ B o is-Reymond 1863. p. 248.

2) $1 . \mathrm{c}$. 
Dr. G. Schwalbe,

Weise zu erklären. Ich fand in anderen Zellen Kerne, die ebenfalls an einer Seite ihren scharfen Contour verloren hatten. Es lag hier aber vor der Oeffnung eine zusammengeballte Masse ungefähr von der Grösse des Kerninhalts, die sich deutlich von der Ganglienzellsubstanz unterschied. Es war hier offenbar der Kern geplatzt und hatte einen Theil seines Inhalts entleert, der hier noch am Kerne liegen geblieben war. Dies gab nun auch den Schlüssel für die Erklärung der eben beschriebenen Bilder. Der Kern war auch dort geplatzt; nur war der herausgetretene Theil des Inhalts hier ganz durch die Zellsubstanz hindurchgetreten und nicht mehr zu sehen (Fig. 5). In einigen Fällen war sogar das Kernkörperchen auf diese Weise verschwunden. (Fig. 4 A vom Maulwurf). Man sieht also, dass Gründe genug vorliegen, an der Deutung jener Bilder als Kernfortsätze zu zweifeln, und bin ich nicht der Einzige, der in der vorliegenden Frage zu negativen Resultaten gekommen ist. So sagt Kölliker, was auf überraschende Weise mit meinen Befunden übereinstimmt, in der neuesten Auflage seiner Gewebelehre p. 331: "Die nähere Prüfung ergab, dass der Nucleus geplatzt war und dass der Nucleolus durch die Substanz der Zelle bis zur Oberfläche sich eine Bahn gegraben hatte, die wie eine vom Kern ausgehende Faser erschien." In gleicher Weise vorsichtig muss man nun meiner Ansicht nach die Bilder, welche einen Zusammenhang zwischen Axencylinder und Kernkör per $\mathrm{ch}$ en demonstriren sollen, auffassen. Haben sich doch auch schon Forscher wie M. Schultze, Leydig, Deiters und Beale in dieser Hinsicht verneinend geäussert. Auch Kölliker, der sich früher nach einigen Beobachtungen am Ganglion Gasseri des Kalbes, woselbst der Nucleolus sich in eine Faser verlängerte, die sich aber nicht in den Fortsatz der Zelle verfolgen liess, dahin zu neigen schien, einen Zusammenhang des Axencylinders mit dem Kernkörperchen für wahrscheinlich zu halten, konnte bei neu aufgenommener Prüfung der Frosch-Ganglienzellen nie wieder etwas Aehnliches sehen ${ }^{1}$ ). Auffallend ist es auch, dass Courvoisier, der doch sonst so genaue Angaben über das Fasernetz der Ganglienzellen macht, die Nervenfaser nur bis a $n$ den Kern verfolgen konnte. Ebenso vorsichtig äussert sich Fraentzel. Ich will endlich noch anführen, dass ich keineswegs Zellen vor mir hatte, in denen der Inhalt des Kernes getrübt oder durch untergelagerte Zell-

1) 1. c. p. 253 . 
substanz undeutlich zu erkennen gewesen wäre; ich hatte im Gegentheil zahlreiche Zellen vor mir, bei denen sich die Substanz des Kernes sehr klar und deutlich zeigte, so dass hindurchziehende Gebilde nicht hätten übersehen werden können.

Ueber die Art und Weise der Verbindung des Axencylinders mit der Ganglienzelle habe ich nun Folgendes beobachtet. Der Axencylinder geht direct in die Substanz derselben über. Wie dies bei den Wirbellosen nicht schwer zu constatiren ist, so auch bei den Wirbelthieren. Der bisher gleichmässig dicke Axencylinder verbreitert sich plötzlich beim Uebergang in die Zellsubstanz kegelförmig, und dieser Kegel geht direct in die feingranulirte Masse der Ganglienzelle über. Man sieht dabei die feine Strichelung des Axencylinders im Kegel divergirend in die Strichelung der Zelle ausstrahlen. Man könnte von einem pinselförmigen Ausstrahlen reden; und scheint es mir, dass die Beobachtung von Sander (Archiv von Reichert und $\mathrm{du}$ Bois-Reymond 1866. p. 401) hierauf zu beziehen ist; nur ist es mir auffallend, dass er dieselbe nur einmal gemacht hat, während diese Verhältnisse doch überall leicht zu constatiren sind. Sehr zu empfehlen sind in dieser Beziehung die Zellen der Spinalganglien der Katze, die sich leicht mit beträchtlich langen Fortsätzen isoliren lassen. Ich gebe in Fig. 3 eine Abbildung einer solchen Zelle, an der namentlich die kegelförmige Verbreiterung der Nervenfaser gut zu erkennen ist. Fig. 1 zeigt dasselbe vom Kalbe (Ueberosmiumsäure-Präparat). Hier ist namentlich die divergirende Strichelung deutlich ausgesprochen.

Eine andere wichtige Frage, die bisher verschieden beantwortet wurde, ist die, wie sich das Nervenmark der zutretenden Faser beim Uebergange in die Ganglienzelle verhält, $o b$ dasselbe, wie A rnold will, in die Zelle hinein zu verfolgen ist und sein Ende im Kern erreicht, ob es bloss bis an die Zelle heranreicht, oder schon früher aufhört. Was zunächst die Angaben Arnold's über eine lichte Markmasse, die in die Zelle hineindringen soll, betrifft, so haben schon andere Forscher genügend ihre Gründe dagegen angeführt. Ich kann noch hinzufügen, dass bei Behandlung der Ganglienzellen mit Ueberosmiumsäure an keiner Stelle der gleichmässig braun erscheinenden Zelle die so charakteristische Färbung des Nervenmarks $\mathrm{zu}$ erkennen ist.

Courvoisier ist der Ansicht, dass bloss blasse Fasern mit einer Ganglienzelle in Verbindung treten, und sah nie ein directes 
Uebergehen markhaltiger Fasern in Zellen ${ }^{1}$ ). Aus seinen Degenerationsversuchen schliesst er sodann, dass die blassen Fasern nicht ganz marklos, sondern nur "markarm « seien.

Gerade in diesem Puncte bin ich nun zu ganz positiven Resultaten gekommen. Ich sah bald, dass sich zur Entscheidung dieser Frage die Ganglienzellen der Säugethiere und Vögel nicht eigneten, da dieselben sich immer ohne Hülle isoliren und dabei vielen Insulten ausgesetzt sind, die zunächst immer auf die Markscheide zerstörend einwirken werden. Ich musste mich desshalb an die Ganglienzellen des Frosches wenden und fand hier in den Zellen der Spinalganglien vorzügliche Objecte, die betreffenden Verhältnisse zu demonstriren, während mir dasselbe an den sympathischen Ganglienzellen nicht gelang. An ersteren, die sich im frischen Zustande leicht mit Hulle isoliren lassen, gelingt es nun häufig, Praparate zu erhalten, an denen man das Nervenmark bis di $\mathrm{cht}$ a n die Zellsubstanz herantreten sieht. Es hört hier die Marksubstanz plötzlich scharf auf, während der Axencylinder auf die oben beschriebene Weise in die Ganglienzelle übergeht. Der Axencylinder wird also gleich bei seinem Hervortreten aus der Zelle von einer vollständigen Markscheide umgeben, so dass man hier direct dunkelrandige Fasern in Ganglienzellen übergehen sieht. Fig. 6 und 7 erläutern die betreffenden Verhältnisse; nur hat sich in Fig. 6 bei der Präparation die Markscheide etwas von der Zelle zuruickgezogen.

Nachdem so das Herantreten der Markscheide bis unmittelbar an die Ganglienzelle unzweifelhaft nachgewiesen worden war, wurden mir auch Bilder verständlich, die ich zuweilen an isolirten Ganglienzellen des Kalbes wahrnahm. Es wird nämlich der Zellfortsatz oft in ganz geringer Entfernung von der Ganglienzelle von einem elliptischen, dunkel und scharf contourirten, stark lichtbrechenden Korn unterbrochen, ans dem dann der Axencylinder unverändert wieder hervortritt. Die Bedeutung dieses Kornes, das nur zuweilen beobachtet wurde, war mir anfangs unklar. Nach dem oben Angeführten kann es aber wohl keinem Zweifel unterliegen, dass wir es hier mit einem zuruckgebliebenen Rest der Markscheide zu thun haben. So wird es denn auch bei den Säugethieren höchst wahrscheinlich, dass die Marksubstanz bis an die Zelle herantritt (vgl. Fig. 2 und 9).

Kürzer kann ich mich über den zweiten Theil der Angaben von

1) 1. c. p. 23 . 
Ueb. d. Bau d Spinalganglien n. Bemerk. ü. d. sympathıschen Ganglienzellen. 69

Arnold fassen, die namentlich durch Courvoisier noch weiter specialisirt sind, nämlich ủber das die Zelle umgebende mit mehreren Fäserchen vom Nucleolus entspringende Fasernetz und über die Spiralfasern, welche sich aus demselben entwickeln. Es wollte mir hier ebenso wenig, wie Kölliker, Fraentzel und Sander gelingen, Fảserchen vom Kernkörperchen ausgehen zu sehen. Was das Fasernetz betrifft, so muss ich entschieden in Abrede stellen, dass ein solches an isolirten Ganglienzellen der Säuger sich findet, seien es spinale oder sympathische Zellen. Es wollte mir hier auch nicht gelingen, Spiralfasern wahrzunehmen. Dagegen erscheinen an den mit Hulle isolirten Nervenzellen des Sympathicus des Frosches da, wo die gerade Nervenfaser abgeht, feine Fäserchen, die dann in eine oder mehrere Spiralfasern ubergehen. Ich habe aber in Betreff des Fasernetzes immer nur solche Bilder erhalten, wie sie Kölliker ${ }^{1}$ ) abbildet. Was die Natur der Spiral fasern betrifft, so bin ich geneigt, zweierlei Arten derselben anzunehmen: 1) nervöse, die unmittelbar aus der Substanz der Zelle entspringen, keine oder nur einige wenige Touren um die gerade Faser machen und sich von dieser nicht wesentlich an Dicke unterscheiden, und 2) Fasern, die als Verdickungen der Scheide aufzufassen sind und sich aus jenem Fasernetz am Grunde der Zelle entwickeln. Im ersteren Falle haben wir es dann mit einer wahren bipolaren Zelle zu thun, deren beide Fortsätze aber nach einer Seite hin verlaufen (vgl. Fig. 182 von Kölliker). Im anderen Falle haben wir dagegen nur wahre unipolare Zellen vor uns, und dieser Fall ist nach meinen Beobachtungen der haufigere.

Erwảhnen will ich endlich noch, dass Sander die Netze an der Oberfläche der Ganglienzellen für Zerklüftungen der Ganglienzellsubstanz, bedingt durch die Einwirkung der Chromsäure hält und Spiralfasern nie gesehen hat. Alleın nach seinen Abbildungen würde wohl Niemand auf die Idee gekommen sein, von Netzen an der Oberfläche der Ganglienzelle zu sprechen. Auch Fraentzel's Erklärung, dass durch das Epithel der Ganglienzelle Fasernetze vorgetäuscht würden, passt nur auf die Säugethiere und Vögel, und will ich auch nicht läugnen, dass ein Theil der Bilder Courvo is i er's sich hierauf zurickführen lasst. Allein auf die Zellen des Froschsympathicus passt jene Erklärung nicht, da wir ja hier von keinem

1) 1. c Fig. 181 p 254. 
epithelialen Belag sprechen können. Worauf hier die Angaben von einem vollständigen Fasernetz beruhen, wage ich nicht zu entscheiden, betone aber nochmals, dass ich, wie Kölliker, nur an der Stelle, wo die gerade Faser sich aus der Zelle entwickelt, Fasergebilde erkennen konnte.

Schliesslich noch einige Bemerkungen über die multipolaren sympathischen Ganglienzellen der Säugethiere. Die Zellen des Sympathicus der Säugethiere unterscheiden sich von denen der Spinalganglien constant durch ihre Multipolarität. So leicht, wie man sich nun hier von uberzeugen kann, so schwierig ist es, die Fortsätze der Zellen weiter zu verfolgen, um etwa eine Verschiedenheit zwischen denselben, entsprechend den ron Deiters an den Zellen des Rückenmarks beschriebenen Verhältnissen, zu constatiren. Nur in einem Falle gelang es mir, eine solche sicher nachzuweisen. Aus dem Sympathicus der Katze isolirte ich die Zelle, welche Fig. 9 ahgebildet ist. Man bemerkt, dass alle Fortsätze, mit Ausnahme eines einzigen, fein granulirt sind und sich bald weiter theilen. Sie wuirden also den "Protoplasmafortsätzen « von De ite r s entsprechen, während der Fortsatz a, welcher sich durch seine geringere gleichmässige Breite und seinen ungetheilten Verlauf von den übrigen wesentli ch unterscheidet als Axencylinderfortsatz aufzufassen wäre. Man bemerkt an ihm überdies ein elliptisches, dunkel und scharf contourirtes Korn, wie ich es schon oben beschrieben und als Rest einer Markscheide gedeutet habe.

Schliesslich sei es mir noch gestattet, an diesem Orte Herrn Professor M. Schultze, der mir bei diesen Untersuchungen stets den freundlichsten Rath und die liberalste Unterstutzung mit literarischen Hülfsmitteln zu Theil werden liess, meinen besten Dank auszusprechen. 


\section{Erklärung der Abbildungen auf Tafel VI.}

Sảmmtliche Figuren von 1 brs 15 sind bei der Vergrosserung System F, Ocular II enes Z ei is'schen Mikroskopes, Fig. 16 bis 20 bei System C, Ocular II desselben Mikroskopes gezeichnet.

Fig. 1. Ganglienzelle aus einem Spinalganglion des Kalbes. Ueberosmiumsäurepràparat. Man erkennt bei a noch Reste des Nervenmarks, be1 $b$ die kegelformige Verbreiterung des Axencylinders.

Fig. 2. Ganglienzelle aus einem Spinalganglion des Kaninchens. In Jodserum frisch untersucht. a Rest des Nervenmarks als scharf contourirtes elliptisches Korn; b wie vorhin.

Fig. 3. Ganglienzelle aus einem Spinalganglion der Katze. Mit zwei Kernkörperehen. Frisch in Jodserum.

Fig 4. Ganglienzellen aus einem Spinalganglion des Maulwurfs. A mit geplatztem Kern ohne Kernkörperchen; B mit drei Vacuolen: a Kern b b b Vacuolen. In Jodserum untersucht.

Fig. 5. Ganglienzelle aus einem Spinalganglion des Kaninchens mit scheinbarem Kernfortsatz. Jodserum.

Fig. 6 und 7. Ganglienzellen ans einem Spinalganglion des Frosches (Rana esculenta.) a Kerne der Scheide, b Pigment. Das Nervenmark tritt in Fig. 7 bis unmittelbar an die Zellsubstanz heran. Jodserum.

Fig. 8. Zelle aus dem Sympathicus von Rana temporaria mit reichlichem Kernlager am Abgang der Nervenfaser. Mittelst der Arnold'schen Methode gewonnen.

Fig. 9. Multipolare Zelle aus dem Sympathicus der Katze. Der Fortsatz a (mit Rest ron Nervenmark) unterscheidet sich wesentlich von den übrigen Fortsåtzen $b$ b.

Fig. 10, 11 und 12. Multipolare zweikernige Ganglienzellen aus den Ganglien des Grenzstranges vom Kaninchen.

Fig. 13. Bipolare einkernige blasse Nervenzellen aus dem Grenzstrange des Kaninchens, welche mit marklosen Nervenfasern in Verbindung stehen. Beim Uebergange einer solchen in die Zelle findet sich immer eine kernhaltige Anschwellung a.

Fig. 14. Multipolare zweikernige Ganglienzelle aus dem Sympathicus des Meerschweinchens. Die Fortsatze sind hier fast alle dicht an der Zelle abgerissen. 
Fig. 15. Ganglienzelle aus dem unteren Schlundganglion von Arion empiricorum mit concentrisch geschichteter Zellsubstanz, grossem Nucleus und acht ausgebildeten Nucleolis, deren jeder eine Vacuole enthált.

Fig. 9 bis 15 frisch in Jodserum untersucht.

Fig. 16. Längsschnitt aus einem Spinalganglion von Rana esculenta. a eintretende sensible Wurzel, b dieselbe, nachdem sie sich am Austritt aus dem Ganglion mit den gangliospinalen Fasern vereinigt hat; c motorische Wurzel. Holzessig-Alkohol-Prảparat.

Fig. 17. Spinalganglion von Lacerta agilis mit verdünnter Kalilauge behandelt. a centraler Theil der sensiblen Wurzel, b dieselbe nach ihrer Vereinigung mit den gangliospinalen Fasern.

Fig. 18. Stückchen eines Spinalganglions vom Kalb mit verdünnter Kalilauge behandelt. a sensıble Fasern, die sich nach der Peripherie zu (b) an die gangliospinalen anlegen.

Fig. 19. Theil eines Querschnitts durch ein Spinalganglion des Kalbes. Holzessig-Alkohol-Praparat. Man erkennt die Vertheilung der durchtretenden Nervenbündel in der Ganglienzellenmasse.

Fig. 20. Dicken-Langsschnitt eines Spinalganglions vom Kalb. Holzessig-Alkohol-Präparat. Es verlaufen hier Nervenfasern in allen Richtungen innerhalb der Schnittebene; ferner siaht man zahlreiche schräg oder querdurchschnittene Nervenfaserbündel. Bindegewebe sehr reichlich entwickelt. 